\title{
Mobilité et apprentissage linguistique : \\ étude du discours métalinguistique d'apprenants Erasmus
}

\section{Introduction}

L'apprentissage linguistique et la notion de mobilité sont intimement liés dès lors que tout apprentissage implique un déplacement de la part du sujet : l'apprenant projette un autre soi dans la langue cible, il se réinvente dans sa relation à l'Autre et à sa langue. De même que tout sujet-locuteur est mobile dans ses imaginaires sur les langues: deux imaginaires au moins se superposent nécessairement, celui de la langue à acquérir et celui (tout aussi fantasmé) de la langue maternelle. Les locuteurs élaborent des représentations à partir de praxis scolaires et sociales, et ces langues « imaginaires » participent de la constitution idéologique de la langue et de ses représentations collectives.

La «mobilité » de l'apprenant a intéressé les didacticiens, mais souvent dans une perspective strictement «additive et monolingue » (Castellotti \& Huver, à paraître) sans tenir compte de l'articulation des savoirs et des compétences dans une perspective inter- ou translinguistique. Nous pensons qu'une vision holistique qui envisage l'apprentissage dans sa globalité serait sans doute plus pertinente. Cela se justifie si l'on pense par exemple au caractère original et non cumulatif de la compétence plurilingue, par définition instable et partielle $^{1}$; à l'articulation complexe des représentations «ordinaires » (culturelles, métalinguistiques) et « savantes » en situation d'apprentissage ; à la trajectoire cognitive ${ }^{2}$ des apprenants confrontés à une réalité langagière et culturelle nouvelle (ex : le développement de la compétence « méta », la dialectique du maintien et de l'abandon des stéréotypes,...).

Dans le cas de la mobilité Erasmus, les étudiants vivent une double expérience qui rend leur apprentissage particulièrement complexe : d'une part celle d'un environnement culturel et linguistique pluriel et instable (F. Dervin parle de « communautés-crochets ${ }^{3}$, ces communautés Erasmus ponctuelles qui se créent et se recréent sans cesse); et d'autre part, celle d'un enseignement basé sur des savoirs présentés comme établis et cloisonnés, souvent éloignés de la réalité langagière et culturelle. M. Anquetil parle de l'expérience Erasmus comme « un moment didactique particulièrement pertinent quant à l'enseignement de la compréhension du monde, de la condition humaine » (2006: 38). Mais qu'en est-il de la formation (méta-) linguistique ?

Nous proposons de nous interroger ici sur les représentations métalinguistiques que les étudiants Erasmus activent ou se forgent dans ce contexte particulier. Plus précisément, quels déplacements catégoriels s'opèrent chez ces étudiants « mobiles » par définition?

Pour répondre à ces questions, nous proposons de pratiquer ce que certains ont choisi d'appeler une « linguistique populaire », en nous intéressant au discours métalinguistique des apprenants. Nous verrons quelles théories spontanées les locuteurs développent sur la langue cible, leur langue maternelle ou les langues véhiculaires qu'ils pratiquent en contexte international. Ces savoirs « ordinaires » ou «profanes» (Beacco, 2004 ; Achard-Bayle \&

\footnotetext{
${ }^{1}$ Coste, Moore, Zarate, 2009 (www.coe.int/t/dg4/linguistic/Source/SourcePublications/CompetencePlurilingue09web_fr.doc)

${ }^{2}$ Nous entendons la dimension cognitive au sens «des processus de construction des connaissances et de leur mise en discours à partir des données reçues par les sens, la mémoire et les relations sociales » (Paveau, $2006: 11$ )

${ }^{3}$ F.Dervin (2009) emprunte l'expression au sociologue Z. Bauman (2004).
} 
Paveau, 2008), se fondent sur des représentations plus ou moins axiologiques, voire un imaginaire linguistique et culturel puriste. Nous analyserons donc la mise en discours de ces représentations afin de voir sous quelle forme émerge la conscience métalinguistique, et comment l'apprentissage est gouverné par des normes diverses, socialement et culturellement construites.

\section{Linguistique populaire et discours métalinguistique}

\subsection{Savoir savant versus savoir ordinaire ?}

Tout locuteur a quelque chose à dire sur sa langue et celle des autres. Selon M.-A. Paveau, ces théorisations métalinguistiques spontanées constitueraient un savoir à part entière qui s'inscrirait dans un continuum. Selon une vision scalaire des théories linguistiques, il y aurait à un extrême les théories des linguistes experts, le savoir savant et légitime, et à l'autre extrême les théories dites «spontanées » ou «populaires » des non-linguistes (2008b : 95). Cette position intégrationniste considère donc les données «folk» comme des données linguistiques, des savoirs subjectifs et incomplets qui devraient être intégrés aux données scientifiques de la linguistique.

Dans une perspective didactique, il s'agirait d'accorder un statut à ce savoir ordinaire qui intervient sur le plan cognitif dans les activités d'enseignement-apprentissage, notamment via l'activité épilinguistique et le discours métalinguistique. A la suite de C. Weber, nous pensons qu'il est important de questionner « la coexistence entre l'oralité ordinaire, scolaire et savante, en tant que ciment des activités sociocognitives. » $(2008: 219)$.

\subsection{Les systèmes de « représentations-connaissances »}

Notre réflexion est didactique au sens où elle relève des « rapports aux savoirs dans des situations d'enseignement et d'apprentissage » (Legardez, 2004 : 648). Nous étudions plus particulièrement les systèmes ou configurations de « représentations-connaissances » en jeu dans le contexte de la mobilité étudiante.

De manière générale, les apprenants sont confrontés à différents genres de savoirs :

- «savoirs de référence » : savants ou scientifiques ;

- « savoirs sociaux» ou «naturels »: les savoirs des acteurs ordinaires (étudiants, enseignants, ...), préalables à l'enseignement ;

- «savoirs scolaires » : construits par les enseignants à partir des savoirs naturels et des savoirs institutionnels de référence (norme prescriptive) et des savoirs « intermédiaires » (manuels, revues pédagogiques...) (ibid. p.648)

Ces systèmes de «représentations-connaissances» seront déterminés par «une matrice culturelle d'interprétation », comprenant certains éléments de la culture nationale, des «pratiques sociales », et enfin les « discours en circulation » (ibid. p.650-651).

Les spécialistes des représentations liées à l'apprentissage linguistique se sont intéressés à la référence stratégique aux représentations en circulation et constitutives d'un imaginaire commun ou d'une mémoire collective, mais aussi à la façon dont ces représentations plus ou moins stables sont activées dans le discours, ou reconfigurées discursivement (Dagenais \& Moore, 2004 : 35). C'est dans cette voie que nous situons notre étude des pratiques métalinguistiques. 


\subsection{Les pratiques métalinguistiques « populaires »}

A la suite d'A. Culioli, nous avons choisi de distinguer l'activité épilinguistique de l'activité métalinguistique: la première n'aurait pas de marques tangibles, alors que la seconde s'exprimerait dans le discours métalinguistique. L'activité épilinguistique serait une activité d'analyse non consciente à laquelle nous soumettons en permanence le discours auquel nous sommes exposés. C'est précisément grâce à cette activité mentale d'analyse que l'enfant acquiert le langage. On ne fait pas que reproduire ou répéter, on analyse, on organise les données langagières. Le fait de tenir un discours sur la langue relèverait d'une activité consciente métalinguistique alors que l'activité épilinguistique serait non consciente et ne génèrerait pas de discours. C'est donc au niveau de la conscientisation que se poserait la différence entre épi- et méta-.

Nous nous intéressons ici au discours métalinguistique, c'est-à-dire aux pratiques réflexives conscientes et rationalisées des sujets dits « ordinaires » (métalangage courant). A la suite de Brekle (1989), nous envisageons deux pratiques métalinguistiques :

1. Théorisations - Descriptions : on décrit l'activité de langage (ex : les catégorisations grammaticales)

2. Prescriptions - Proscriptions : on prescrit/proscrit les comportements langagiers (ex : ça se dit/ ça ne se dit pas)

Ces pratiques métalinguistiques vont se manifester discursivement sous différents traits. Nous nous intéresserons aux suivants :

1. Les subjectivèmes (Kerbrat-Orrechioni, 1980):

a. Les déictiques : surtout l'alternance pronominale qui nous informe sur le positionnement énonciatif des sujets.

b. Les modalisations: verbes, adjectifs, adverbes évaluatifs modalisateurs (du type vrailfaux) et axiologiques (du type bon/mauvais).

2. Les organisateurs textuels-cognitifs (Paveau, 2006) :

a. La typologie : elle permet de catégoriser les objets du monde et opère un classement plus ou moins axiologique et stéréotypé des objets linguistiques et des locuteurs.

b. La métaphore : elle sert d'organisateur cognitif, discursif et textuel. «La métaphore, comme concept mental, appartient en effet au stock prédiscursif des locuteurs, et prend sa source dans leur conceptualisation expérientielle du monde. Elle fonde les structures sémantiques sur le vécu expérientiel des sujets (...) $\gg$ (ibid. p.198).

c. L'antithèse : notamment dans l'élaboration des auto- et hétéro-stéréotypes.

\subsection{Norme(s) et stéréotypes}

Les représentations imaginaires, prescriptives, de toute langue ont ce rôle : assigner un lieu, fixe, immuable, à la langue, la belle, la vraie (...) Et voilà pourquoi l'on vous coupe ou vous reprend au nom d'un savoir qui n'est que faux semblant linguistique et vraie prescription (une norme fictive : la belle langue ou une norme prescriptive : la vraie langue) 
A.-M. Houdebine, «Langue et imaginaire: le français aujourd'hui », Salon du livre, Genève, mai 1988.

Le discours métalinguistique spontané de nos étudiants se caractérise par une fluctuation entre différentes normes subjectives (fictives, prescriptives, communicationnelles) (Houdebine, 2002) et l'alternance entre des positionnements « homogénéisant » (marqués par une distanciation et le retranchement derrière une certaine doxa) versus " hétérogénéisant » (marqués par l'implication personnelle). Comme nous le verrons, cette dynamique attitudinale correspond à une certaine mise en scène identitaire des sujets qui naviguent entre différentes représentations, normes et stéréotypes.

D'après Oesch-Serra et Py, la situation du voyageur est marquée par la «mise en échec de ses schèmes d'interprétation de la vie quotidienne » (1997: 31). Or, le stéréotype serait un genre de discours particulier qui permet de donner du sens de façon économique et automatique à une nouvelle réalité, et d'assurer un consensus social au sein de la nouvelle communauté qui se crée sur base de convictions qui servent de nouveaux modèles et référents.

On verra dans notre corpus que les étudiants ont recours à la fois à des formules stéréotypées «élémentaires » et à de nouvelles formules «élaborées » en fonction du contexte. On aura par exemple des stéréotypes bipolaires, qui mettent en relation d'opposition communautés d'origine et d'accueil:

- des hétéro-stéréotypes: l'objet du stéréotype ne coïncide pas avec la communauté d'appartenance du locuteur

- des auto-stéréotypes: l'objet du stéréotype coïncide avec la communauté d'appartenance du locuteur.

\section{Corpus et méthodologie}

Les extraits d'entretien présentés ici proviennent du corpus constitué dans le cadre de notre recherche doctorale. Nous avons mené des entretiens individuels semi-directifs avec vingt étudiants Erasmus non-francophones séjournant à Liège. Les entretiens (entre 15 et 45 minutes selon les participants) ont été enregistrés et transcrits. Nous avons choisi une transcription orthographique standard inspirée des conventions de transcription proposées par le groupe VALIBEL de l'UCL et par l'Université Paul-Valéry Montpellier $3^{4}$.

Dans le cadre de cet article, nous avons choisi de nous attarder sur trois entretiens, dont deux ont été réalisés avec $\mathrm{M}$ (étudiante polonaise biologiste) au début et à la fin de son séjour, et le troisième avec $\mathrm{K}$ (étudiante italienne linguiste) au début de son séjour. Nous tenons à préciser que nos analyses visent à donner un premier aperçu des mécanismes qui sous-tendent la pratique métalinguistique spontanée dont notre thèse de doctorat rendra compte de manière plus exhaustive.

\section{Les pratiques métalinguistiques Erasmus : une cartographie stéréotypée}

Les acteurs sociaux ont recours à des schémas discursifs stéréotypés qui vont leur permettre d'organiser les perceptions de leur nouvel environnement. Chez nos étudiants,

\footnotetext{
${ }^{4}$ Les conventions sont disponibles sur Internet : http://asl.univ-montp3.fr/UE11/norme.pdf et http://www.uclouvain.be/cps/ucl/doc/valibel/documents/conventions valibel 2004.PDF
} 
plusieurs normes identitaires et normes d'identification ${ }^{5}$ sont en concurrence, comme cet extrait l'illustre :

3E- qu'est-ce que vous avez observé comme différences ?

4K- l'intérêt dans les études et le comportement de la gens de la Belgique. parce qu'ils sont très gentils et très intelligents et ils ont beaucoup de respect pour les études et pour les autres gens d'autres pays et pour moi c'est très important. en Italie il n'y a pas beaucoup de gens qui- ouverts, ils sont un peu égoïstes et ils ne me plaît pas. et en Italie il n'y a pas de gens qui aiment étudier, faire des recherches, essayer, travailler à beaucoup de choses. ils ne l'aiment pas. moi je suis très dynamique et je dois faire beaucoup de choses et je suis très curieuse ici c'est l'ambiance idéale pour moi.

Dès le début de l'entretien, l'étudiante (désormais K) stigmatise les membres de sa communauté d'origine. Elle élabore des représentations contrastives selon le principe organisateur de l'antithèse. En effet, selon une dialectique sociale qui oppose le «moi » et l'« autre », K exprime ses convictions par des stéréotypes bipolaires via une énonciation explicite. La nouvelle formule associe des hétéro-stéréotypes (les Belges sont "gentils», « intelligents », studieux et travailleurs) à des auto-stéréotypes (« en Italie... » les gens sont «égoïstes », peu studieux et fainéants). On remarque que certaines des propriétés appliquées aux Belges et aux Italiens ne relèvent pas de représentations collectives. En effet si la gentillesse et le caractère ouvert des Belges appartiennent à un univers de discours connu, l'égoïsme et la fainéantise des Italiens relèvent moins du domaine de l'évidence. La nouvelle formule ne semble donc productive que dans le contexte spécifique de la mobilité étudiante. On n'observe cependant qu'une seule marque de modalisation du prédicat (dans « ils sont un peu égoïstes ») susceptible de confirmer le caractère limité de la formule. Dans l'ensemble, on a une formulation assertive négative généralisante («il n’y a pas »), excepté à la fin du tour où l'énonciateur refait son apparition avec un ancrage déictique explicite («moi je»). L'énonciateur se positionne explicitement en opposition vis-à-vis de sa communauté d'origine en se qualifiant de «très dynamique », très active ( « je dois faire beaucoup de choses ») et de «très curieuse ». Elle termine par signaler implicitement son identification au groupe d'accueil (« ici c'est l'ambiance idéale pour moi »).

Par la suite, $\mathrm{K}$ active une série de stéréotypes linguistiques qui contribuent à la mise à distance de sa communauté d'origine. On assiste à l'élaboration d'une typologie axiologisée qui participe d'une dialectique, linguistique celle-là. Ce deuxième système est mis en relation avec le système dialectique social décrit supra via la pratique de la stigmatisation, «l'acte de stigmatisation qui transfère sur la langue l'opposition sociale » (Delveroudi \& Moschonas, 2008 : 15). Ce double mouvement, à la fois linguistique et social, traduit plus largement une attitude puriste via un discours esthétique et idéologique qui qualifie et disqualifie en fonction de critères comme la beauté, la clarté etc. et qui fait appel aux affects. (Paveau \& Rosier, 2008 : 52)

28E- quelle langue est-ce que vous préférez ?

29K- j'aime bien le français mais je ne sais pas si je le préfère parce que l'anglais c'est pour moi c'est la meilleure. si tu as besoin d'exprimer un concept, je pense que c'est plus facile. aussi parce qu'il n'est pas très défini la signification des mots. et tu peux donner beaucoup de significations à ta parole.

30E- vous vous sentez bilingue?

$31 \mathrm{~K}$ - je suis bilingue parce que je suis née en Albanie. je suis déjà bilingue. avec l'italien et l'albanais. je connais 5 langues.

\footnotetext{
${ }^{5}$ Laurence Brunet-Hunault (1996) distingue les normes identitaires (l'image langagière que le groupe impose) et les normes identificatoires (l'image que donne aux autres la langue qu'on parle). Ces deux images langagières révèlent le double rôle de la langue dans la construction identitaire des sujets.
} 
32E- et comment vous vous sentez dans chaque langue ? c'est différent ?

$33 \mathrm{~K}$ - oui très différent. [...] la structure de ces langues c'est très différente. l'albanais c'est une langue très particulière, l'italien c'est plus douce. l'anglais c'est la plus facile mais aussi la plus expressive. le français c'est comme la musique, c'est comme une musique et aussi comme la mathématique. il y a beaucoup de choses, des exceptions, des choses particulières que tu dois mémoriser mais c'est un plaisir de parler en français. l'allemand c'est très compliqué. c'est très compliqué mais c'est aussi très intelligent. c'est une langue intelligente parce que la structure, la composition c'est élaboré et significatif. c'est fort. j'ai étudié un peu de russe la première année d'université et je l'aime bien. je pense que c'est ma langue préférée, avec l'anglais. l'anglais et le russe.

34E- pas l'italien.

$35 \mathrm{~K}$ - l'italien $\mathrm{mm} /$ je ne sais pas. c'est différent. c'est très différent. j'aime l'italien mais c'est une autre chose. ce n'est pas une langue germanique. la structure de la langue germanique je pense que c'est un chose plus plus intéressant et plus élaboré. l'italien c'est difficile la grammaire est très difficile mais je pense qu'il manque quelque chose c'est un problème de culture je pense. dans la Russie, dans l'allemand, dans l'anglais tu peux trouver un autre univers et pour moi c'est très charmant. je suis allée c'était le *sweden* et c'est le pays que j'aime plus.

Différents axes d'évaluation se dessinent :

- cognitif (langue conceptuelle, facile, élaborée, structurée,...)

- $\quad$ expressif (langue expressive,...)

- $\quad$ esthétique (langue musicale, douce, belle, charmante,...)

On assiste à un double mouvement de catégorisation et d'essentialisation, deux processus cognitifs en jeu dans le processus représentationnel métalinguistique (Paveau, 2010). K construit une série d'ontotypes ${ }^{6}$ (la structure de la langue germanique, la difficulté de la grammaire italienne, la musicalité du français etc.). Elle qualifie les langues d' « intelligentes », d' « élaborées » de « significatives ».

Les locuteurs ont en effet recours à des catégories qui permettent de distinguer, désigner, classer les langues d'après leurs usages et leurs fonctions (ex: langue véhiculaire), des catégories qui acquièrent le statut de caractéristiques intrinsèques et naturelles des langues. Or, on sait que les langues envisagées dans la variété de leurs emplois ne correspondent pas toujours aux représentations et aux discours essentialistes en circulation dans l'imaginaire collectif. En effet, le français en particulier serait traversé par une double tension: «une tendance centripète qui le ramène à lui-même et à sa perfection imaginaire, et une tendance centrifuge qui le dote d'un expansionnisme au statut mixte entre culture et communication » (ibid. p.28).

Notre extrait illustre la dimension puriste de la représentation métalinguistique décrite supra. Les langues sont évaluées selon des critères subjectifs mais qui font appel à des cadres prédiscursifs collectifs (Paveau, 2006). La stigmatisation de certains traits définitoires des langues participe de l'élaboration d'un imaginaire linguistique collectif. On notera que les axes d'évaluation divergent selon les familles de langues : axes cognitif et expressif pour les langues germaniques versus axe esthétique pour les langues romanes.

Depuis le début de l'entretien, K se positionne en porte-à-faux vis-à-vis de sa communauté d'origine. Les hétéro-stéréotypes linguistiques qu'elle élabore sur les langues germaniques activent en filigrane une série symétrique d'auto-stéréotypes sur l'italien et plus largement les langues romanes. Le fait de relever les qualités intrinsèques de certaines langues souligne implicitement que ces qualités font défaut aux autres langues. Ce discours métalinguistique rejoint une tendance générale chez l'étudiante à se distinguer des membres

\footnotetext{
${ }^{6}$ A côté des sociotypes, ethnotypes, Rosier et Ernotte (2004) proposent la catégorie des ontotypes qui attribuent à une caractéristique un statut ontologique ( les ontotypes se présentent comme des évidences anthropologiques »p.39)
} 
de sa communauté d'origine (italienne) telle qu'elle se la représente, et à s'identifier plutôt comme une citoyenne européenne. On voit donc s'élaborer au fil de l'entretien une série de représentations socio-langagières qui font écho à certaines idées doxiques liées au projet de création d'une grande «société cognitive européenne» (ex : la valorisation d'une langue véhiculaire commune - l'anglais lingua franca - vs la promotion du plurilinguisme européen). Et ce discours politiquement correct est régulièrement mis à mal par le recours à des stéréotypes linguistiques et plus largement à des évaluations axiologisées explicites sur les qualités intrinsèques des différentes langues européennes.

Nos représentations des langues sont donc constituées d'un « déjà-là » cognitif, une sorte de «mémoire de la langue » qui organise nos perceptions. Cependant, et l'analyse de notre corpus l'atteste, les représentations initiales s'enrichissent aussi de l'expérience individuelle. Celle-ci participe de l'élaboration contextuelle de représentations plus personnalisées qui se traduisent par l'élaboration de nouvelles formules plus ou moins stéréotypées.

L'extrait d'entretien qui suit témoigne de ce processus de catégorisation et d'élaboration de la représentation à partir de connaissances empiriques. M a été interrogée à la fin de son séjour :

23E- c'est quoi la langue française pour toi ?

24M- mais ça c'est la liberté ! [rires] oui vous m'avez posé cette question au début / ben c'était moi qui a choisi cette langue quand j'étais plus petite et en plus c'est un choix pour être plus indépendante parce que chez moi j'ai quatre frères je suis donc, et toute ma famille parle en anglais parle en allemand / donc moi j'ai dit je veux pas que les autres (...) et j'ai dit je veux pas qu'ils m'aident je veux être-créer mon monde propre et donc pour ça je choisis le français je pense et voilà / [...]

$25 \mathrm{E}$ - et c'est quoi ce monde?

26M- je trouve que moi je suis une autre personne quand je parle en français / ben je suis plus plus courageuse, plus indépendante, ça me donne beaucoup de liberté je peux choisir tout ce que je veux je peux faire tout ce que je veux parce qu'il n'y a personne qui peut influencer mes décisions [...]

$27 \mathrm{E}-\mathrm{il}$ y a d'autres choses qui sont différentes quand tu parles français ?

28M- ben je pense que je suis moins timide quand je parle en français / (...) avec la langue française c'était toujours bien donc euh tout ce que j'ai fait avec ça ça finit bien donc voilà j'ai beaucoup de souvenirs de bons souvenirs.

L'étudiante recourt dans un premier temps à une métaphore : «ça c'est la liberté !». Pour que la métaphore fonctionne, elle doit faire appel à des cadres collectifs d'interprétation qui relèvent de la mémoire cognitivo-discursive des différents interlocuteurs. On y voit un appel à la devise nationale française «Liberté, Egalité, Fraternité » à laquelle l'étudiante fait écho à d'autres moments de l'entretien lorsqu'elle parle de l'histoire nationale française. Cela fait également appel au discours déjà ancien sur le français «langue d'ouverture », «langue libérale » :

«Que de choses éternellement bonnes et vraies ont été pour la première fois dites en français, ont été frappées en français, ont fait leur apparition dans le monde en français. Que d'idées libérales et justes ont trouvé tout d'abord en français leur formule (...). C'est une langue libérale vraiment » (E. Renan, 1888)

Cet effet d'interdiscours témoigne de l'existence de cadres prédiscursifs partagés par l'étudiante.

Par ailleurs, la métaphore renvoie à l'expérience personnelle de $\mathrm{M}$ pour qui les caractéristiques intrinsèques à la langue deviennent des traits définitoires de son identité. Parler français est synonyme de révolution personnelle, d'indépendance. Les qualités de la langue cible deviennent les siennes. Sa description spontanée met en exergue la dimension 
performative de la langue, où parler revient à agir « je suis une autre personne quand je parle français / ... je peux faire tout ce que je veux ». Ce n'est pas une identification «plurilingue » qui se profile, mais plutôt deux identités séparées, deux monolinguismes. Le courage et l'indépendance sont nettement associés à la langue française, comme si ces traits ne pouvaient pas se manifester lorsqu'elle parle sa langue maternelle. La langue française n'est plus seulement celle de l'Autre, elle fait partie de l'identité langagière du sujet qui attribue à la langue des changements de comportement connotés positivement.

De la même façon, les extraits qui suivent montrent la trajectoire de l'étudiante dans l'élaboration de ses théorisations langagières. À son arrivée à Liège, M catégorise les « français » qu'elle a rencontrés :

21E- et tu penses que tu as besoin d'un professeur pour apprendre le français ici ? [...]

22M- oui oui. je parle beaucoup avec les amis, les collègues avec qui je partage (...) mais c'est vrai qu'ils parlent très très vite et parfois on se (...) bien alors je sais qu'il faut essayer un petit peu parler avec le prof qui parle très bien en français qui n'utilise pas de mots qui sont bon euh de la vie quotidienne, des abréviations et tout ça / et je trouve que c'est mieux parler- oui penser beaucoup, éviter les erreurs pour [interruption extérieure] euh bon euh j'ai fini bon qu'il faut- oui qu'il faut toujours euh /// que je dois éviter les erreurs que je normalement je parle avec les Belges- il me comprend mais quand même c'est je je voudrais parler bien en français pas comme justement pour parler $(. .)-$. pour parler bien. alors j'ai besoin de cours ici.

Spontanément elle souligne la spécificité discursive du parler «populaire » ou « familier » qu'elle oppose au parler « soutenu » de l'enseignant ( «le prof qui parle très bien en français qui n'utilise pas de mots qui sont (...) de la vie quotidienne, des abréviations »). Elle produit une petite sociologie spontanée sous forme de typologie axiologisée des parlers. Le discours de l'enseignant est valorisé. Elle mentionne les «erreurs » qu'il faut «éviter » quand on parle avec des francophones. La conscience de l'erreur l'emporte sur la conscience de la compétence ${ }^{7}$. Même si la norme communicationnelle est préservée, c'est la norme linguistique qui prévaut, et celle-là s'acquiert nécessairement en contexte d'apprentissage guidé. Le locuteur natif constitue la référence :

31E- et tu préfères parler français avec des francophones ou avec des non francophones ?

32M- j'ai toujours de la chance [rires] de parler en français avec les francophones (E- oui ?) oui je je oui- bon je cherche pas toujours les gens qui parlent en français, les gens francophones mais bon je parle toujours- je préfère parler avec des gens francophones.

33E- pourquoi ?

34M- bon [rires] pour apprendre la langue, la langue CORRECTE et aussi l'accent

Il maîtrise « la langue correcte » et « l'accent ». La représentation est homogène, au détriment de la variation socio-langagière.

Par opposition au français du natif, $M$ décrit une autre pratique du français comme langue véhiculaire inter-Erasmus :

27E- et le français que tu parles avec les erasmus ?

28M- oui le français que je parle avec les erasmus c'est le même français que je connais [rires]

La question induit la catégorisation, mais M lui reconnait spontanément une spécificité: «c'est le même français que je connais ». Se dessine donc une communauté de locuteurs qui partageraient une même variété de langue.

\footnotetext{
${ }^{7}$ Voir l'étude de M. Stegu et H. Wochele sur la linguistic awareness (2008).
} 
29E- et eux ?

30M- et eux- bon mais eux- bon ils parlent en français bien sûr mais je pense qu'ils sont aussi le même problème avec la grammaire avec la prononciation comme moi alors

Le français pratiqué par les étudiants Erasmus est considéré comme problématique. La difficulté se situe au niveau grammatical et phonétique.

Enfin, si l'on s'intéresse au discours de $\mathrm{M}$ à la fin de son séjour, on constate que la position très homogénéisante du début évolue vers une représentation plus hétérogène :

15E- et qu'est-ce que tu dirais du français que les étudiants Erasmus parlent entre eux ?

$16 \mathrm{M}$ - ben c'est le français littéraire [rires] parce que ben on connaît pas beaucoup de mots familiers donc on utilise $\mathrm{mm}$ voilà / on fait beaucoup l'attention pour parler bien pour utiliser les schémas de grammaire parfaite de subjonctif et tout ça [rires] mais normalement quand je parle avec les Français et les Belges ben j'ai jamais entendu qu'ils parlent si, je sais pas, correctement que nous / oui mais en plus les étrangers quand ils parlent en français il y a toujours une petite comment dire une petite / ben si on comprend pas on peut utiliser le mot en anglais par exemple / ou on dit en notre langue maternelle et c'est pourquoi c'est un petit peu bizarre / en plus il y a beaucoup de gens qui parlent en franco-anglais c'est-à-dire tout ensemble [rires] et comme ça ils font / je sais pas c'est difficile de comprendre mais quand même il faut essayer /// oui mais c'est surtout la différence c'est quand on utilise les mots vraiment beaux- beaux mots.

Au fil de son discours, $\mathrm{M}$ renverse la formule élémentaire : «parler bien » relève de la compétence du non-natif et non plus du natif. Le non-natif serait davantage attentif à la correction de la langue, "les schémas de grammaire parfaite du subjonctif », alors que «normalement (...) j'ai jamais entendu qu'ils parlent si (...) correctement que nous ». Le français pratiqué par les allophones serait le «français littéraire», considéré comme le français correct. Le positionnement métalinguistique est plus hétérogénéisant et pose une question «socialement vive » (Legardez): c'est quoi la correction finalement ?

M élabore un auto-stéréotype relatif à la communauté d'appartenance Erasmus (les Erasmus parlent «le français littéraire »). L'alternance pronominale est significative du point de vue de l'identification du sujet aux différents groupes de locuteurs. Alors que dans le cadre du premier entretien, $M$ utilisait les pronoms "eux" et "ils" pour désigner les autres étudiants Erasmus et se distinguait de ce groupe par l'opposition au "moi", à présent l'étudiante utilise le pronom «on» («on connaît pas beaucoup de mots familiers », « on fait beaucoup l'attention $», \ldots$ ) et dès lors s'identifie comme membre de la communauté «mobile». L'étudiante oscille entre différents positionnements énonciatifs. Elle commence en s'identifiant comme locuteur «Erasmus » («on» = nous les étudiants Erasmus), elle donne donc une image assez solide d'elle-même et de son groupe, opposé au groupe, lui aussi homogène, des Français et des Belges. Ensuite elle passe au « je », l'énonciateur assume son propos et modalise davantage, ce qu'elle avance n'est pas systématiquement vrai et elle émet une réserve sur la valeur de vérité de l'énoncé. Elle modalise avec un complément assertif: «normalement», et elle situe : «quand je parle avec les français ». M évalue donc ce qu'elle va dire comme étant approximatif: «je sais pas » exprime le doute. Après s'être présentée comme appartenant au groupe de locuteurs Erasmus, elle s'en distancie (« les étrangers quand ils parlent $\gg$ ) pour mieux s'y rallier lorsqu'il s'agit de justifier par l'exemple. Elle se distancie de nouveau lorsqu'elle parle des pratiques plurilingues ( il y a beaucoup de gens qui parlent en franco-anglais »). Enfin, du point de vue de l'élaboration de la formule stéréotypée, on observe de nombreuses traces de modalisation : « une petite », «c'est un petit peu bizarre ». On voit bien ce que Oesch-Serra et Py appellent une « dialectique du maintien et de l'abandon 
du stéréotype » due au fait que les représentations sont mises à mal par l'expérience empirique des sujets.

On devine donc la concurrence de normes identificatoires et identitaires à travers l'usage des marqueurs énonciatifs déictiques, et l'alternance d'auto- et d'hétéro-stéréotypes selon les pratiques dont on parle. Le processus de catégorisation complexe qui sous-tend la mise en discours se veut le miroir des identifications plurielles du sujet.

\section{Conclusion}

Nous avons tenté de caractériser les pratiques métalinguistiques des étudiants Erasmus afin de voir comment émerge leur sentiment linguistique (déterminant pour l'apprentissage de la langue cible), et sous quelle forme se développe leur conscience métalinguistique dans le contexte d'apprentissage complexe de l'Erasmus.

D'abord, notre analyse a bien montré le caractère à la fois cognitif, discursif et social des représentations métalinguistiques. Selon un double mouvement de catégorisation et d'essentialisation, les sujets classent les langues, les locuteurs, leurs parlers et leurs accents. On voit se développer une double dialectique, à la fois sociale et linguistique, qui se réalise dans l'acte de stigmatisation. Les sujets ont recours à des formules stéréotypées «élémentaires » ou «élaborées » en rapport avec le contexte social, de même qu'ils font appel à différents organisateurs textuels-cognitifs tels que la typologie, la métaphore ou l'antithèse pour structurer leurs représentations.

Mais même si ces catégorisations font régulièrement appel à des cadres prédiscursifs collectifs, elles ne sont pas toujours figées. En effet les étudiants «mobiles » présentent une certaine «mobilité » dans leurs imaginaires. Notre étude comparative de deux entretiens effectués à cinq mois d'intervalle confirme cette hypothèse. On constate par exemple que les représentations des pratiques en français langue véhiculaire se construisent au fur et à mesure que les apprenants sont confrontés à de nouvelles données empiriques liées au contexte Erasmus. Mais dans le même temps, ces représentations s'élaborent par rapport à des représentations collectives sur la correction de la langue et le modèle du locuteur natif qui relèveraient d'une sorte de «matrice culturelle d'interprétation » commune (Legardez). Notre analyse montre que la représentation de la correction de la langue semble mise à mal par l'expérience du sujet qui est forcé de constater que les francophones n'emploient pas toujours les formes prescrites par la grammaire normative ou qu'ils n'ont pas recours au modèle du «bon français standard » tel que les non-francophones peuvent se le représenter.

On peut donc avancer que la conscience normative de nos étudiants Erasmus serait plutôt axée sur la norme prescriptive et l'idéal de la correction de la langue (entendue comme la maîtrise d'un registre de langue soutenu), plutôt que sur la maîtrise des normes pragmatiques et des conventions discursives. Même si le sujet admet que les pratiques langagières entre non-natifs «fonctionnent» et permettent (voire facilitent) la communication, les conventions qui caractérisent ces nouvelles pratiques discursives ne semblent pas tout à fait admises et légitimes. De même, alors que les pratiques discursives des natifs ne correspondent pas à la représentation de la norme linguistique et à la langue apprise en classe de FLE dans le pays d'origine des apprenants, ces nouvelles données semblent difficiles à intégrer aux anciens schèmes d'interprétation de la réalité discursive.

À partir de ces observations, il s'agirait à présent de réfléchir à intégrer les pratiques métalinguistiques des apprenants Erasmus dans le cadre de l'enseignement-apprentissage linguistico-culturel. Que ce soit par une préparation à la rencontre interculturelle qui partirait 
des représentations des acteurs concernés, mais aussi une réflexion sur les pratiques correctives et la place de la norme dans l'apprentissage.

\section{Pour citer cet article :}

Référence : Meunier, Deborah (2011), «Mobilité et apprentissage linguistique : étude du discours métalinguistique d'apprenants Erasmus », Etudes de Linguistique Appliquée, 162, p.137-151.

\section{Références bibliographiques}

ACHARD-BAYLE, G., PAVEAU, M.-A. 2008. «La linguistique « hors du temple »?», Pratiques, 139-140, p. 3-16.

ANQUETIL, M. 2006. Mobilité Erasmus et communication interculturelle. Peter Lang.

AUGER, N., et al. 2008. Pour une didactique des imaginaires dans l'enseignementapprentissage des langues étrangères. L'Harmattan.

BEACCO, J.-C. 2004. «Représentations métalinguistiques ordinaires et discours », Langages, 154. Paris : Larousse.

BREKLE, H. E. 1989. «La linguistique populaire », dans S. Auroux (dir.), Histoire des idées linguistiques. Bruxelles : Mardaga, tome 1, p. 39-44.

BRUNET-HUNAULT, L. 1996. "Imaginaire linguistique, enquête auprès d'étudiants de Deug 1 de psychologie ». Travaux de linguistique, 7, Université d'Angers, p. 37-42.

CASTELLOTTI, V., HUVER, E. 2012 (à paraître). « Mobilités et circulations académiques : dynamiques, catégorisations, évaluations ou «Bougez, il en restera toujours quelque chose »», Le Discours et la Langue, E.M.E.

COSTE, D., MOORE, D., ZARATE, G. 2009. Compétence plurilingue et pluriculturelle.

Conseil de l'Europe. Disponible sur Internet :

www.coe.int/t/dg4/linguistic/Source/SourcePublications/CompetencePlurilingue09web_fr.doc CULIOLI, A. 1997. "Subjectivité, invariance et déploiement des formes dans la construction des représentations linguistiques », in FUCHS, C. et ROBERT, S. (éds). Diversité des langues et représentations cognitives. Paris, Ophrys, Collection «L'homme dans la langue », p. 43-57. DAGENAIS, D., MOORE, D. 2004. «Représentations ordinaires du plurilinguisme, transmission des langues et apprentissages chez des enfants, en France et au Canada ». Langages, 154, p. 34-46.

DEFAYS, J.-M. 2012 (à paraître). «La mobilité étudiante en question : la perspective des linguistes et des didacticiens », Le Discours et la Langue, E.M.E.

DEL VEROUDI, R., MOSCHONAS, S. 2003. «Le purisme de la langue et la langue du purisme », PhiN, 24, p. 1-26.

DERVIN, F. 2008. Métamorphoses identitaires en situation de mobilité. Turku : Presses universitaires de Turku.

ERNOTTE, P., ROSIER L. 2004. «L'ontotype : une sous-catégorie pertinente pour classer les insultes? », Langue française, 144, p. 35-48.

HOUDEBINE, A.-M. 2002. L'imaginaire linguistique. L'Harmattan.

KERBRAT-ORECCHIONI, C. 1980. L'énonciation. Paris : Armand Colin.

MEUNIER, D. 2010. «De la «mobilité » de l'imaginaire linguistique chez les étudiants Erasmus », Synergies Pays Riverains de la Baltique, 7, p. 61-76.

LEGARDEZ, A. 2004. "L'utilisation de l'analyse des représentations sociales dans une perspective didactique : l'exemple de questions économiques », Revue des sciences de l'éducation, vol. 30, 3, p. 647-665. 
OESCH-SERRA C., PY, B. 1997. «Le crépuscule des lieux communs, ou les stéréotypes entre consensus, certitude et doute », Tranel, 27, p. 29-49.

PAVEAU, M.-A 2006. Les prédiscours. Sens, mémoire, cognition. Presses Sorbonne Nouvelle.

PAVEAU, M.-A., ROSIER, L. 2008a. La langue française. Passions et polémiques. Vuibert. PAVEAU, M.-A. 2008b. «Les non-linguistes font-ils de la linguistique ? Une approche antiéliminativiste des théories folk », Pratiques, 139-140, p. 93-109.

RENAN, E. 1888. «Extrait d'une conférence faite à l'Alliance pour la propagation de la langue française ». Disponible sur Internet :

http://agora.qc.ca/francophonie.nsf/Documents/Histoire de la langue francaise--

La_langue de_la_liberte_par_Ernest_Renan

STEGU, M., WOCHELE, H. 2008. «Le français langue internationale: normes et implications didactiques », Synergies Europe, 3, p. 113-125.

VON MÜNCHOW, P. 2010. «Langue, discours, culture : quelle articulation ? (1ère partie) », Signes, Discours et Sociétés [en ligne], 4. Disponible sur Internet : http://revuesignes.info/document.php?id=1439

WEBER, C. 2008. «Les verbalisations ordinaires dans la classe : objets furtifs ou variables encombrantes des sciences du langage ? », Pratiques, 139-140, p. 219-237. 